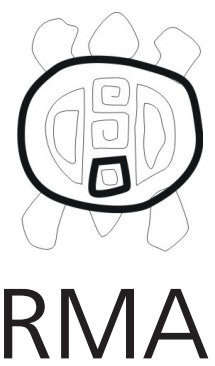

Dossier

\section{Neoevolucionismo y ecología cultural. La obra de Julian Steward y la renovación de la enseñanza de la antropología en la Argentina}

Gastón Julián Gil

CONICET-Universidad Nacional de Mar del Plata. E-mail: gasgil@mdp.edu.ar

\begin{abstract}
Resumen
La introducción de la obra del antropólogo norteamericano Julian Haynes Steward (1902-1972) produjo una parcial renovación de la enseñanza de la antropología en la Argentina desde mediados de la década del cincuenta. Principalmente en arqueología (y más tarde en lo que comenzaría a llamarse antropología social), el enfoque materialista, neoevolucionista y ecológico entró en tensión con la corriente histórico-cultural alemana que había hegemonizado el campo antropológico nacional liderado por el italiano José Imbelloni desde la Universidad de Buenos Aires. La circulación de estas nuevas ideas -que también provocaron fuertes polémicas en su ámbito de gestación original- es abordada principalmente a partir de la labor transformadora y revitalizadora de Alberto Rex González, posgraduado en la Universidad de Columbia y alumno de Steward a fines de los años cuarenta. De este modo, se propone un análisis de las maneras en que nuevos postulados conceptuales provenientes de una tradición metropolitana se difundieron en un contexto periférico.
\end{abstract}

Palabras Clave: Antropología argentina; Neoevolucionismo; Ecología cultural; Universidad

Neo-evolutionism and cultural ecology. The Argentine Renovation of Anthropology Teaching under the influence of Julian Steward

\begin{abstract}
The introduction of the U.S. anthropologist Julian Haynes Steward (1902-1972) to the Argentine courses of Anthropology in the mid-50's, launched a process of partial renovation in Argentine anthropology. His materialistic, neoevolutionist, and ecological scope applied to Archeology and, later, to what would become Social Anthropology, differed from more established views such as German historical-cultural theory led by Italian anthropologist José Imbelloni and Austrian archaeologist Oswald Menghin. This paper describes and analyzes the arrival of Steward's ideas to some Argentine schools of Anthropology (Córdoba, Litoral/Rosario and La Plata) in the late 40s, by means of archaeologist Alberto Rex González, a Columbia Ph.D. and one of Steward's students. In fact, studying Steward's diffusion in Argentina may cast some light upon the ways in which concepts and theoretical frameworks born in metropolitan anthropologies, reach peripheral contexts and are transformed within their new academic homes.
\end{abstract}

Keywords: Argentine anthropology; Neoevolutionism; Cultural Ecology; University

\section{Encuentros y tensiones teóricas en la antropología argentina}

La circulación local desde la década de 1950 de la obra del antropólogo norteamericano Julian Haynes Steward (1902-1972) fue una de las instancias a partir de la cual la hegemonía de la corriente histórico-cultural alemana en la Argentina comenzó a ser discutida. Como consecuencia, se generarían importantes tensiones que darían lugar al desarrollo de otra concepción de esta ciencia y en particular de la subdisciplina arqueológica, pero que también abonarían el terreno para el desarrollo de la antropología social desde los años sesenta. En su contexto periférico, la antropología argentina -liderada hasta mediados del siglo XX por el italiano José Imbelloni desde la Universidad de Buenos Aires-, reproducía los patrones historicistas que habían caracterizado una tradición metropolitana (Cardoso de Oliveira 1995) -la alemana- que se encontraba en franca retirada en el escenario antropológico mundial. Esta corriente históricocultural rechazaba, a grandes rasgos, al evolucionismo y a las antropologías que "han tomado del lenguaje sociológico algo más que el lenguaje" (Imbelloni 1935: 209), en alusión a la antropología social británica. Imbelloni fustigaba a la antropología social por nutrirse de una matriz limitada "por un pensamiento de extrema relatividad" (Imbelloni 1935: 209). Al confiar en las fortalezas de una aproximación inductiva y filosófica frente a la concepción "práctica" y "normativa", condenaba aquellos intentos de priorizar "la morfología interna de una cultura" (Imbelloni 1935: 209) que apenas reproduce "fórmulas caducas y cambiantes" (Imbelloni 
1935: 209) y que persigue la armonía de los componentes internos. Con la definitiva institucionalización de la disciplina que se concretó a partir de la creación de las carreras de grado, en las universidades nacionales de La Plata y Buenos Aires, estos enfoques difusionistas dominantes hegemonizaron de igual modo las currículas y no permitieron la generación de espacios para el desarrollo de otras concepciones teórico-metodológicas que caracterizaban los estilos disciplinares de las tradiciones metropolitanas norteamericana, británica y francesa. De esa manera, la circulación de autores y teorías que excedieran el mainstream difusionista fueron gestándose en espacios marginales de esas carreras, en otros espacios institucionales y a partir de la labor de investigación, docencia y formación de recursos humanos de personajes que, con mayor o menor capacidad de liderazgo, pudieron construir sus trayectorias académicas, también con impacto diverso.

Como indica Altamirano para toda América Latina, no es ninguna novedad que "nuestros países ingresaron con retraso al mundo moderno y culturalmente continuaron desempeñando el papel de provincias de las grandes metrópolis, sobre todo de las europeas, que funcionaban como focos de creación y prestigio de donde provenían las ideas y los estilos inspiradores" (Altamirano 2008: 10). Sin embargo, el modo en que las teorías circulan en un campo disciplinar desde una tradición metropolitana a otra periférica merece un análisis detallado. Se trata de una clase de intercambio internacional que, como "los textos no importan su contexto con ellos" (Bourdieu, 2000: 161), abre un amplio espacio para una reinterpretación "en función de la estructura del campo de recepción" (Bourdieu, 2000: 161). En efecto, las maneras en que circulan las ideas (en este caso, por ejemplo, como postulados teóricos) dan cuenta de las apropiaciones locales de ciertos autores, de sus posiciones en las luchas de poder dentro de un campo, de la conformación de liderazgos, del nacimiento de líneas de investigación y el establecimiento de genealogías académicas, explícitas e invisibles (Darnell 2001) y "linajes ocultos" (Guber 2006). $Y$ por supuesto, también se pueden leer - a la luz de esa circulación de ideas- cómo interactúan las nuevas perspectivas analíticas con los cambios institucionales, principalmente en lo que hace a la consolidación de nuevas redes académicas emergentes con capacidad de producir alteraciones en el equilibrio de fuerzas de un determinado campo disciplinar. En relación a ello, Bourdieu entiende que la estructura del campo universitario obedece a las relaciones de fuerzas que existen entre los agentes que dan cuenta de un estado en un momento temporal determinado. Esos agentes están dotados de cuotas de poder que "detentan a título personal y sobre todo a través de las instituciones de las que forman parte: la posición ocupada en esta estructura está en el principio de las estrategias que apuntan a transformarla o a conservarla modificando o manteniendo la fuerza relativa de los diferentes poderes
0 , si se prefiere, las equivalencias establecidas entre las diferentes especies de capital" (Bourdieu 2008:171). Pero además, las transformaciones globales que se puedan suscitar en el campo global (por ejemplo el aumento de la matrícula en un determinado momento político, el aumento presupuestario para investigación, fundación de nuevas universidades) se traducen en cambios morfológicos evidentes que redundan en una modificación "de la relación de fuerzas entre las facultades y las disciplinas, y sobre todo, dentro de cada una de ellas, entre los diferentes grados" (Bourdieu 2008:171-172). Por ello, se hace necesaria una "historia estructural" del campo universitario.

Como señalan Darnell y Murray (2004) cuando en los años sesenta se comenzó a delinear en los Estados Unidos la especialidad de historia de la antropología, se gestó "una dialéctica de praxis y reflexividad, una reinvención continua" (Darnell y Murray 2004: xiii) de la disciplina. En ese contexto, los mismos autores afirman que se cristalizó una "retórica de la discontinuidad" que caracteriza a la antropología norteamericana, a partir de la cual el legado boasiano se invisibilizó y varias de las nociones centrales de su obra (el antirracismo, el relativismo cultural, la idea de pluralidad) se incorporaron al sentido común de la disciplina, naturalizándose y olvidando quiénes fueron sus promotores. De ese modo, se plasmarían "genealogías invisibles" que están encarnadas en las ideas, los contactos personales y los acuerdos institucionales que son fundamentales para definir una identidad disciplinaria, en este caso la antropología norteamericana. Eso le permitió entonces a las críticas feminista, posmoderna y poscolonial -principalmente desde sus inquietudes por la narrativa y el diálogo- proclamar arrogantemente (Darnell y Murray 2004.) su autonomía y la inexistencia de vínculos con el pasado. La propia Darnell sostiene además que si bien los antropólogos suelen situarse en genealogías exclusivas, sus propias prácticas denotan otro tipo de influencias y linajes mucho más complejos que los que conscientemente proclaman (Darnell y Murray 2004).

\section{Julian Steward y la antropología norteamericana}

La historia de la antropología norteamericana difícilmente pueda comprenderse en forma cabal sin considerar en detalle la figura de Julian Steward (1902-1972), fundador de lo que él mismo denominó "ecología cultural" y partidario de -también en sus propios términos- un evolucionismo multilinear. Steward protagonizó -según Marvin Harris (1997)- junto con Leslie White (19001975) y el arqueólogo australiano Vere Gordon Childe (1892-1957), la "restauración nomotética", orientada a reconducir los enfoques particularistas que, desde la descollante labor de Franz Boas y sus discípulos, habían hegemonizado el campo de la antropología cultural en los Estados Unidos. Steward concibió un proyecto intelectual integrador de la disciplina antropológica que involucraba principalmente a la arqueología y a la 
antropología social, pero también a una antropología aplicada y de las sociedades complejas, "modernas", o "nacionales", un término que utilizaba corrientemente. Esa poderosa impronta que dejó en una de las más importantes tradiciones antropológicas metropolitanas también se explica a partir de los vínculos y la influencia que ejerció durante su labor docente en la Universidad de Columbia. En su relativamente breve paso entre 1946 y 1952 por aquella "torre de marfil" todavía identificada con la figura de Boas, estudiantes que luego se transformarían en destacados exponentes de la antropología norteamericana se nuclearon en torno a su liderazgo intelectual. Antropólogos de la talla de Eric Wolf, Sidney Mintz, Roy Rappaport, Morton Fried, John Murra, Elman Service, Andrew Vayda, Robert Murphy y Robert Manners encontraron en Steward una atención especial durante sus seminarios pero sobre todo un enfoque materialista que contrastaba notoriamente con la escuela de Cultura y Personalidad que encarnaba la otra figura polar del departamento: Ruth Benedict. Esos estudiantes conformaron informalmente el Mundial Upheaval Society (MUS) denominación irónica de connotaciones artísticas (upheaval significa trastorno). Según Peace (2008) este grupo presenta una importancia notoria para entender la historia de la antropología en los Estados Unidos y las tensiones sociopolíticas dentro de la academia antes y después del Macartismo. Los integrantes del MUS, cuyos vínculos personales y académicos perduraron en el tiempo, eran mayormente militantes de partidos de izquierda (aunque Steward siempre se mantuvo alejado de la política) y rechazaron el relativismo y el particularismo dominantes en su medio, características del legado boasiano. De ese modo, abrazaron las posturas neoevolucionistas y materialistas de autores como el propio Steward y los mencionados Leslie White y Gordon Childe, a quienes nunca interpretaron como antagonistas. Procedían mayormente de los estratos medios y mediosbajos, y habían accedido a la Universidad de Columbia gracias al GI Bill, un subsidio que le permitió a los veteranos de guerra acceder a estudios de postgrado que de otra manera difícilmente habrían podido concretar. Una parte importante de los más notables antropólogos norteamericanos (Clifford Geertz, Marshall Sahlins, entre otros, además de los mencionados de Columbia) fue beneficiaria de ese subsidio oficial.

Con la llegada de Steward a Columbia, no pocos estudiantes -sobre todo los militantes de izquierda- se vieron atraídos por el apasionamiento en sus clases y la posibilidad de trabajar con ejemplos contemporáneos y polémicos, como considerar a la Revolución China como un caso de cambio evolutivo independiente (Peace 2008). Además, Steward se involucró personalmente con la formación y el futuro académico de sus tesistas, muchos de los cuales, por ejemplo, obtendrían plazas de profesores temporarios en la Universidad de Illinois -su destino a partir de 1952- cuando escaseaban los puestos de profesores. El otro de los ejes que parece haber ejercido una notoria influencia fue la importancia que Steward le estaba empezando a asignar al estudio antropológico de las sociedades contemporáneas, enfatizando la especificidad antropológica frente a la sociología en el estudio de los mundos modernos. Entre aquellos jóvenes estudiantes de Columbia se encontraba un médico argentino que sería una figura clave en la renovación del campo arqueológico nacional desde los años cincuenta y que tendría una labor relevante como docente en la formación de muchos futuros antropólogos sociales que transitaban la universidad argentina en la década del sesenta: Alberto Rex González.

\section{Rex González y la antropología argentina}

De acuerdo con los datos (testimonios y documentos como programas de materias) con los que se cuenta y las fuentes indirectas, la tarea docente de Alberto Rex González (n. 1918) en el marco de sus cátedras, resultó vital para la difusión de nuevos autores que escapaban a la lógica dominante de la corriente histórico-cultural alemana que emanaba desde el Museo Etnográfico en la Universidad de Buenos Aires. Pero además de lo meramente formal de sus cátedras, es necesario destacar la labor de formación plasmada en los grupos de investigación que se construyeron alrededor suyo y, sobre todo, en los espacios de cierta informalidad a partir de su liderazgo carismático, entendido como una dimensión del poder, traducido bajo "otro nombre de la legitimidad, producto del reconocimiento, del desconocimiento, de la creencia «en virtud de la cual las personas que ejercen autoridad son dotadas de prestigio»" (Bourdieu 2007: 227).

A su regreso de los Estados Unidos, los métodos empleados por González entraron en tensión con los postulados aceptados mayoritariamente en el campo antropológico argentino. Principalmente la utilización sistemática de la estratigrafía y el empleo "revolucionario" del fechado radiocarbónico, configuraron "una nueva etapa para la arqueología en la Argentina" (Pérez Gollán 1998: 18), que empezaría a incorporar preocupaciones y conceptos de la corriente histórico-cultural norteamericana (Politis 2001), como el de área cultural, las tipologías y las influencias ambientales. Rex González consiguió realizar en el laboratorio de la Universidad de Yale en los Estados Unidos el primer fechado radiocarbónico del país, referido en este caso a las grutas de Intihuasi, pudiendo establecer así una antigüedad en 6000 A.C. Este procedimiento posibilitó, a modo de experimento crucial, construir las primeras cronologías absolutas en la Argentina, "hito que marca la extinción definitiva de toda especulación cronológica. La consecuencia más directa es que no quedó ni el rastro de la certeza de Boman (a la que muchos adherían con absoluta fe ciega) de que las poblaciones indígenas del NOA, apenas eran un par de siglos más antiguas que la invasión europea" (Pérez Gollán 1998:19). Más tarde, ya como miembro activo de las distintas comisiones del CONICET, consiguió el apoyo 
de este organismo de investigación del estado argentino creado en 1958 para montar el primer laboratorio en el país donde se desarrollara la técnica del fechado radiocarbónico, para la Universidad Nacional de La Plata. Ello permitió, por ejemplo, aplicar para la región andina "un modelo diacrónico ${ }^{1}$ de gran profundidad histórica para interpretar el desarrollo de las sociedades indígenas del NOA" (Pérez Gollán 1998: 18). De esa forma, se comenzó a trabajar sobre cronologías culturales, principalmente basadas en un modelo de periodización propuesto por Wendell Bennett (Bennett et al. 1948) para esa región, pero influenciando notoriamente a otras áreas culturales, "no sólo por un injerencia directa en la discusión sobre unas y otras sociedades, sino por la penetración teórico-metodológica que ejerce sobre equipos diversos de investigadores que se desempeñan en todo el país" (Boschin 1998: 12). Sobre estos nuevos parámetros, los grupos de investigación que se formaron en torno a la figura de González emprendieron trabajos de campaña que privilegiaban las labores de excavación y que además contemplaban la incorporación de estudiantes y jóvenes graduados inclinados hacia la antropología social. En relación a ello, como señalan aquellos jóvenes (dedicados a la arqueología pero también a la antropología social) que no estaban ligados (ni como alumnos en instituciones o como parte de sus grupos de investigación) formalmente a González, lo reconocen como un referente indiscutible. Así, puede aparecer como el "maestro dedicado a despejar nuestros interrogantes" (Boschin 1998: 13), o como quien les mostró "el concepto de antropología como totalidad pero, por sobre todo, un ejemplo ético que nunca dejaremos perder y que deberá orientar toda nuestra tarea profesional" (Ratier 1998: 45). En la misma sintonía, la figura de González ha sido definida como "el constante recordatorio de que había otra antropología posible" (Llamazares 1998: 61), además de considerarlo como "un "atractor pregnante", como un "maestro remoto" (Llamazares 1998: 61). De un modo similar, quienes sí fueron sus alumnos y optaron por la antropología social, también lo señalan como un referente central en las inicios de la joven subdisciplina, en un marco sociohistórico en el que la "historia fundante es una historia de pequeños grupos, de personalismos que canalizaron el sentido social del período" (Ringuelet 1998: 47). De hecho, fue José Cruz, ${ }^{2}$ discípulo de González, graduado en la carrera de historia

\footnotetext{
${ }^{1}$ Carbonari y Figini (1998) destacan en la obra de González la calidad de los estudios diacrónicos, contextuales (no tipológicos) como un intento explicativo de los procesos culturales.

${ }^{2}$ Cruz dejó su cargo tras la Noche de los Bastones Largos en 1966 y se radicó en la ciudad venezolana de Mérida. Su lugar fue ocupado Mario Margulis, un entonces joven contador público y graduado de la especialización en sociología de la Universidad de Buenos Aires, que luego establecería una larga y sostenida trayectoria en el campo de las ciencias sociales argentinas (exilio mexicano mediante durante el Proceso militar de 1976) que se prolonga hasta la actualidad. Su llegada a La Plata resultó problemática para aquellos ayudantes alumnos que completaban la cátedra de Antropología Social, algunos de los cuales fueron reemplazados en sus funciones docentes en las cátedras, según relatan los implicados, por diferencias políticas.
}

de la Universidad de Córdoba, quien se hizo cargo de la cátedra de antropología social, materia optativa del plan naturalista y arqueológico del Museo de la Universidad Nacional de Plata.

Ese vínculo entre las subdisciplinas antropológicas se sostendría en una obra enfocada a mostrar que es "posible buscar una interpretación integral de la cultura sin perder por ello el rigor en el nivel más básico de descripción arqueológica o desatender el desarrollo de técnicas refinadas de recopilación e interpretación del material de campo tanto propio como ajeno" (Ceballos e Hidalgo 1998: 19). Las mismas autoras señalan que González desarrolló un proyecto "ambicioso" que giró en torno a tres dimensiones fundamentales: la relevancia de la investigación empírica; la utilización de nuevos y más rigurosos métodos y tecnologías; y "la articulación en explicaciones abarcativas y unificadas teóricamente" (Ceballos e Hidalgo 1998: 20). Todo ello en pos de lograr una síntesis entre "una aproximación descriptiva (clasificaciones, tipologías, seriaciones) y una aproximación interpretativa o explicativa de escenarios históricos" (Ceballos e Hidalgo 1998: 20). Precisamente, uno de los tantos "alumnos informales" de González, destaca en relación a su afiliación teórica adquirida en los Estados Unidos que el neoevolucionismo "prestaba atención a los procesos actuales. La evolución no había cesado o amenguado y el interés por lo contemporáneo se extendía a sociedades campesinas o aún urbanas" (Ratier 1998: 49). Del mismo modo, Ratier considera que aquello que en los años sesenta se denominó antropología social por sus primeros cultores fue antes que nada una "actitud hacia el estudio del presente y las pretensiones de aplicabilidad del conocimiento eran la frontera. En Buenos Aires dimos en llamar antropología social a aquella que privilegiaba la problemática actual y aspiraba a intervenir en la resolución de problemas" (Ratier 1998: 43).

Aunque para muchos arqueólogos no están demasiado claros los fundamentos neoevolucionistas en gran parte de los trabajos de González, nunca dejó de afirmar que su búsqueda analítica fue siempre la "evolución cultural". Mientras que, por ejemplo, Márquez Miranda daba cuenta de una homogeneidad socio-cultural de las poblaciones de los Valles Calchaquíes a los que se denominaba "diaguitas" desde la conquista española, González:

"se esforzó por identificar una pluralidad de adaptaciones culturales locales en una dinámica cultural regional más amplia que comprendía al noroeste argentino inscripta junto con sus áreas aledañas, señalando que era un craso error metodológico no sólo atribuir homogeneidad a esas culturas, sino presuponer la existencia necesaria de cualquier continuidad cultural entre las poblaciones prehispánicas y las que hallaron los conquistadores españoles en el siglo XVI" (ver Soprano en este volumen). 
Aquí la noción de área cultural cumplía un papel relevante en las interpretaciones, descripciones y clasificaciones, ya que se ponía el énfasis en la relación de las respectivas poblaciones con su medio ambiente, consideradas más directas en aquellas culturas de menor desarrollo (González y Pérez 1972). Ello no excluía, la existencia de un gran centro de irradiación de civilización: "el "Centro Nuclear Andino".

De cualquier manera, mucho tiempo después de su formación en Columbia, González seguía confiando en que "la idea de Evolución y la explicación de sus mecanismos es uno de los objetivos fundamentales de la ciencia" (González 1998: 364). Así, le adjudicaba a la complejidad de la cultura la imposibilidad de haber encontrado todavía explicaciones análogas a las de evolución biológica. Entonces, abogaba por la formulación de explicaciones evolutivas de la cultura que contemplen todos los subsistemas de ese proceso de cambio constante en el tiempo hacia formas más complejas. Al considerar al proceso evolutivo de la cultura como un "hecho incontrovertible" (González 1998: 364) postulaba la utilización de un enfoque descriptivo (cómo se desarrolló la evolución) del que deben hacerse cargo la prehistoria y la arqueología, y el restante explicativo (por qué ocurrió la evolución) tarea de la antropología social y cultural (González 1998: 364). De cualquier modo, González (1974) había insistido en desligar cualquier tipo de relación mecánica entre el desarrollo tecnológico y sus aplicaciones prácticas, ya que "los sistemas simbólicos pueden intervenir y llegar a jugar un rol decisivo. Pero esto no significa que no exista otro proceso paralelo e independiente que origine el cambio a partir de inventos o creaciones cuyo origen sea directamente el de llenar necesidades prácticas inmediatas" (González 1998: 369). Por consiguiente, "el proceso de Evolución Cultural estaría basado en la interacción permanente entre los sistemas simbólicos y los tecnológicos, junto a los biodemográficos. Actuando en conjunto como un todo frente a los procesos ecológicos de adaptación al medio natural como al cultural" (González 1998: 372).

\section{Steward y las corrientes histórico-culturales}

Tanto Steward como White (aunque con diferentes enfoques) recuperaron en la antropología social los aportes de los principales autores evolucionistas del siglo XIX, con particular énfasis en el legado de Lewis Henry Morgan pero también del británico Edward Tylor. Aunque ambos estaban influenciados por el marxismo, discreparon notoriamente en la clase de generalización que postulaban (de mayor alcance en White), además de que el trabajo de Steward era bastante más sensible a los cambios no evolutivos y a las especificidades de cada cultura. Steward colocó un énfasis marcado en las determinaciones ecológicas, a diferencia de White que estaba más interesado en la producción energética de cada cultura. La adopción de un enfoque neoevolucionista fue sistemática durante toda su trayectoria intelectual pero se concretó progresivamente, ya que se formó como antropólogo en un medio en donde primaban los enfoques histórico-culturales que ponían un énfasis en los fenómenos de difusión. Tras graduarse en zoología y geología en la Universidad de Cornell, Steward ingresó con 23 años en 1925 en la Universidad de California, Berkeley, cuyo departamento de antropología estaba conducido por dos de los primeros y más famosos boasianos: Alfred Kroeber (1876-1960) y Robert Lowie (1883-1957). Pese a la resistencia que Steward iría evidenciando con los postulados teóricos de sus profesores y que eran ampliamente aceptados en el campo disciplinar de la época en los Estados Unidos, los trabajos en el marco de su posgrado respetarían a grandes rasgos el clima de ideas y enfoques dominantes. Kroeber y Lowie mantenían además estrechos contactos con los difusionistas alemanes. Uno de sus principales referentes, Wilhelm Schmidt (1868-1954), pasó un semestre en Berkeley desarrollando una intensa actividad académica e interactuó de forma fluida con los estudiantes, inclusive con el mismo Steward.

Cuando su obra alcanzó la madurez, Steward planteó explícitamente la contradicción entre las aproximaciones históricas, particularistas, frente a los planteos "científicos" que buscan generalizaciones, a los que él adhería. En sus propias palabras, la ciencia antropológica debería "intentar clasificar fenómenos en categorías ordenadas, para reconocer las consistentes interrelaciones entre ellas, y así establecer leyes o regularidades, y hacer formulaciones que tengan un valor predictivo" (1972: 3). De esa manera, consideraba que el concepto de difusión había impedido la consideración de las relaciones de causa y efecto en la explicación de la historia cultural. De acuerdo con Steward, los postulados difusionistas acerca del origen de los rasgos culturales no alcanzan a explicar nada, ya que la "difusión se vuelve una causa mecánica e ininteligible, aunque universal, y es utilizada en contraste con otras clases de causas para dar cuenta de casi el 90\% de la cultura mundial" (Steward 1972: 182).

Steward abogaba por explicaciones de otro tipo que revelaran el modo en que se aceptan determinados rasgos culturales que, en definitiva, constituyen particularismos que distinguen las sociedades pero obscurecen las regularidades. Como consecuencia, las regularidades culturales se pueden formular en diversos niveles, cada uno de ellos en sus propios términos. A la difusión sólo le corresponden los aspectos secundarios de la cultura, y no los del núcleo cultural, es decir "los tipos básicos de los patrones sociales, económicos y religiosos" (Steward 1972: 208). De esta manera, concedía que es posible encontrar fenómenos de difusión incluso entre hemisferios pero de ningún modo como primera explicación del desarrollo cultural, que es más bien el producto de la interrelación funcional de los patrones de subsistencia y asentamiento, la población, la estructura 
social, el trabajo cooperativo, la guerra y la religión. Todos ellos tienen sus formas distintivas y requieren formulaciones especiales. De esa manera, Steward proponía una metodología que fuera capaz de determinar regularidades de forma, función y procesos que ocurren transculturalmente entre sociedades que se encuentran en áreas geográficas diversas.

Steward aclaraba que la ecología cultural no era una teoría sino un método, el cual consistía en que "las regularidades transculturales que surgen de procesos adaptativos similares en condiciones ambientales también similares son funcionales o sincrónicos por naturaleza" (Steward 1972: 5). Así, suponía que los cambios culturales apuntan a una mayor complejidad cultural, por lo que el evolucionismo multilinear es básicamente una metodología que supone que se producen regularidades significativas en el cambio cultural y que están determinadas por leyes culturales. De este modo, Steward era partidario de un evolucionismo que buscara formas recurrentes, procesos y funciones antes que esquemas universales omniabarcativos. Las taxonomías se construyen entonces a partir de las diversas clases de paralelismos y similitudes que existen en las diversas culturas pero que se distinguen por su limitada ocurrencia y especificidad. Así, le interesaban los términos sociológicos (banda, clan, clase, estado) que se usan para describir características que se encuentran repetidamente en culturas que no han tenido contacto probado. Allí surge la importancia del concepto de tipo cultural que se diferencia de área cultural en varios aspectos. En principio, se trata de características seleccionadas y no de un contenido total de elementos. Lo que se hace es seleccionar constelaciones especiales de rasgos causalmente interrelacionados que se encuentren entre dos o más culturas, pero no necesariamente en todas. Pero esa selección de características debe realizarse en función del enfoque y del problema, por lo que cualquier aspecto de la cultura puede adquirir una importancia primordial. A diferencia del concepto de tipo cultural, la noción de área cultural supone ciertas similitudes conductuales que se dan en regiones con uniformidades ambientales. La cultura sería entonces una especie de ajuste a las presiones ambientales particulares, aunque Steward admitía que "varios patrones diferentes pueden existir en cualquier área y que diferentes culturas pueden existir en similares condiciones ambientales" (Steward 1972: 35).

\section{Steward y el estudio de los mundos contemporáneos}

A finales de la década del 30, Steward había abandonado sus estudios sobre los nativos norteamericanos y se encaminó a organizar estudios enfocados regionalmente hacia Latinoamérica, estimulado por la inquietud de generar conocimiento y formulaciones teóricas universalmente válidas para la comprensión del cambio cultural. Patterson y Lauria-Perricelli (1999) aseguran que se inspiró en dos investigaciones a las que ayudó a organizar: el Proyecto Virú Valley (desarrollado por William Strong, Gordon Willey y Wendell Bennett), y el Proyecto Yucatán (financiado por la Carnegie Foundation y liderado por Robert Redfield). Ello coincidió con un enfoque estratégico de las agencias oficiales norteamericanas hacia América Latina, acompañado por el interés de las fundaciones filantrópicas. De hecho, hacia mediados de la década del 30 comenzaron a ser financiadas actividades orientadas a ese subcontinente, tanto por organismos oficiales como el Social Science Research Council (SSRC) como por las fundaciones Guggenheim, Carnegie y Rockefeller. Con el ingreso de los Estados Unidos en la Segunda Guerra Mundial, se intensificaron además los programas de entrenamiento en lenguas extranjeras, en un marco general en el que las universidades fueron puestas al servicio de los esfuerzos bélicos. La Fundación Rockefeller ocupó una posición clave en el financiamiento de esas actividades desde 1940 -incluso antes de desatarse la guerra- cuando bajo la tutela de Nelson Rockefeller, el gobierno federal había creado la Office of the Coordinator of Interamerican Affairs (OCIAA), la cual proveyó el financiamiento de la mayor parte de las iniciativas académicas hacia América Latina (Patterson y Lauria-Perricelli 1999: 221).

Principalmente a partir del proyecto Puerto Rico (19481949), Julian Steward se vinculó de un modo directo con una antropología de las sociedades complejas, o más bien de las "sociedades nacionales" como solía denominarlas. El Proyecto Puerto Rico fue financiado por la Fundación Rockefeller con la intención declarada de contribuir a la formulación de planes de desarrollo que favorecieran la transformación industrial y la estabilización política. Ello encuadraba perfectamente en las inclinaciones de Steward hacia cierto desarrollismo, dado que confiaba en que las sociedades marchaban hacia una sucesión de formas más complejas y emergentes. Así, planteaba -a partir también de los hallazgos de Redfield en Yucatánque la integración podía producirse con la urbanización y el abandono de los patrones folk culturales y sociales pero también con la incorporación al estado nacional de todas aquellas subculturas especializadas regionalmente que no se hayan urbanizado completamente y que se han readaptado antes que transformado. De todos modos, también concebía que la transición de lo tradicional a lo moderno produce una serie de conflictos -principalmente generacionales- entre los patrones de cooperación y compartición más tempranos y los objetivos de maximizar el beneficio económico (Clemmer y Myers 1999). En el estudio de lo contemporáneo Steward se vio atraído por la oposición folk-urbano de Robert Redfield (1897-1958), ya que consideraba que las culturas tribales presentaban características similares a las folk: pequeñas, aisladas, homogéneas, cerradas, con patrones que giran en torno al parentesco, orientadas hacia objetivos y valores e impregnadas "por un supernaturalismo general" (Steward 1972: 53). Siguiendo también la lógica de Redfield, consideraba que las sociedades en proceso 
de urbanización se caracterizaban por estar altamente desorganizadas a partir de un rápido cambio que las llevaba hacia la secularización, la individualización y la primacía del conocimiento científico.

A diferencia de las sociedades tribales o "primitivas" caracterizadas por la homogeneidad, el escaso margen de desviación y el alto nivel de integración, Steward aseguraba que las sociedades nacionales no pueden pensarse sobre la base de patrones culturales porque se carece de esas uniformidades en la conducta individual. En contraposición, optó por analizar el "carácter nacional" a partir de los niveles de integración sociocultural, como un común denominador de la conducta que puede verse afectado por factores tales como la crianza en el seno de la familia, las presiones institucionales (principalmente las escuelas) y los medios de comunicación de masas. En lo que consideraba una marcada heterogeneidad de las sociedades modernas, Steward proponía el concepto de niveles de integración sociocultural como una herramienta metodológica capaz de dar cuenta de los diversos grados de complejidad de las culturas, ya que nos provee "un nuevo marco de referencia y un nuevo significado al concepto de patrón; y facilita la comparación transcultural" (Steward 1972: 52). Según Wolf, el concepto de niveles de integración sociocultural constituye un intento de "generar una construcción teórica para entender el microcosmos estudiado en un contexto más amplio" (2006: 28), y que en el marco de la idea de evolución emergente, "tuvo por objeto sugerir que las unidades de la misma clase, cuando se hallan sujetas a procesos integrativos, podrían dar unidades nuevas que no solamente incluyeran las del nivel inferior sino que también mostraran características cualitativamente diferentes en el nivel superior, emergente" (Wolf 2006: 29). También Wolf señala que Steward "usó el concepto para rebatir argumentos que trataban a «la comunidad» como una pequeña reproducción de «la nación», como si se tratara de fenómenos estructurales cualitativamente idénticos. Sin embargo, enseguida procedió a erguir un edificio conceptual en el cual las unidades del nivel familiar se convertían en partes de un nivel regional, y en que las unidades del nivel regional se volvían partes del nivel de la nación" (Wolf 2006: 28-29).

\section{Steward en la antropología argentina}

La obra de Steward fue introducida en la Argentina casi de un modo contemporáneo con sus trabajos y sus textos estuvieron presentes en las carreras de antropología de Buenos Aires y La Plata y en carreras de historia como Rosario y Córdoba. Además, la figura de Steward gozaba de una presencia relevante en América Latina debido a sus tareas como editor del Handbook of South American Indians, gestado en el marco de Bureau of American Ethnography (BAE) del Instituto Smithsoniano. El Handbook había sido una idea original de Robert Lowie pero se desechó en los difíciles tiempos de la depresión económica que siguió al crack de Wall Street en 1929. Cuando el proyecto se reflotó en 1939 el BAE aprobó el financiamiento y le encomendó a Steward una tarea que iba necesitar de un gran esfuerzo colectivo. La edición del Handbook fue un trabajo de largo aliento para el que Steward debió realizar diversos viajes a Sudamérica, entre ellos la Argentina, ${ }^{3}$ a cuya capital consideró como "un sumamente animado centro de civilización" (Kerns 2003: 227). Este ambicioso proyecto se encuadró perfectamente con su idea cada vez más sólida de estudiar en su totalidad las diferentes culturas humanas, de las más "simples" a las más "complejas". Allí, pudo capitalizar provechosamente la tarea de más de 80 académicos, la mayoría de ellos arqueólogos y etnógrafos respaldados por trabajos de campo en las diversas sociedades sudamericanas consideradas. A partir de su rol de editor, pudo imponer los formatos de los artículos, instruir a los autores para que cubrieran las temáticas sobre la base de un orden y criterio determinados. Como señala Kerns (2003), en todo el Handbook sobrevuela implícitamente la distinción analítica entre el núcleo cultural y los rasgos culturales secundarios. Por eso, el sumario destaca cuestiones tales como los patrones de asentamiento, la tecnología, la economía y las formas de organización social y política. Por el contrario, quedaron relegados aspectos tales como los ciclos vitales, mitología, religión y folklore. Steward utilizó una serie de cuatro tipos culturales ("The Marginal Tribes", "The Andean Civilizations", "The Tropical Forest Peoples" y "The Circum-Caribbean Peoples") para organizar el Handbook y presentar de forma ordenada una cantidad tan grande de información histórica, arqueológica y etnográfica, basada en el criterio de que los datos se organizaran que categorías que tuvieran un significado real e histórico (Patterson y Lauria-Perricelli 1999). Cada tipo se basó en la interpretación de aspectos tales como las prácticas económicas, la organización sociopolítica y las actividades rituales antes que en elementos sociales o históricos contingentes que producen la diversidad.

Los autores argentinos que participaron del Handbook fueron Fernando Márquez Miranda ("The Diaguita of Argentina" y "The Chaco-Santiagueño culture", en volumen 2), Salvador Canals Frau ("The Huarpe", volumen $1 \mathrm{y}$ "Expansion of the Araucanians in Argentina", volumen 2), Antonio Serrano ("The Charrua" y "The Sambaquís of the Brazilean Coast", volumen 1), Eduardo Casanova ("The cultures of the Puna and the Quebrada de Humahuaca", volumen 2), Francisco De Aparicio ("The Comechingón and their neighbors of the Sierras de Córdoba", volumen 2), Joaquín Frenguelli ("The present status of theories concerning the Primitive Man in Argentina") y José Imbelloni ("Chefalic Deformations of the Indians in Aregntina", volumen 6). Márquez Miranda, que definía como "monumental" al Handbook, destacaba que a partir de esa publicación Steward "arriba al establecimiento de

\footnotetext{
3 . Según sugiere Kerns (2003) uno de los principales problemas que Steward tuvo con los antropólogos argentinos fue que algunos de ellos no entregaron sus contribuciones a tiempo.
} 
áreas que participan de las características socio-políticoreligiosas, triple basamento que, cuando se logra, puede dar mejor el cuadro general de las regiones culturales en esta parte meridional del Continente americano" (Márquez Miranda 1958: 29). Incluso se refería a Steward como "mi amigo" (Márquez Miranda 1958: 29), más allá de que comentara con cierto escepticismo -dada su adhesión al método histórico-cultural- sobre el "inesperado retorno hacia el evolucionismo" (Márquez Miranda 1958: 35) del que realiza un análisis algo esquemático e incompleto. De todos modos consideraba que "Steward encara de una manera inédita el problema del desarrollo cultural. Los autores anteriores -durante el medio siglo transcurrido- sostienen o demuestran, en su casi totalidad, la imposibilidad de formular leyes que esclarezcan el desarrollo de la cultura" (Márquez Miranda 1958: 35).

En la difusión de la obra de Steward ocupó un lugar privilegiado el arqueólogo Alberto Rex González, quien -como ya se ha mencionado- formó parte del primer grupo de alumnos de Steward en Columbia. Su llegada a esa universidad se vio facilitada gracias al contacto que el arqueólogo Antonio Serrano ${ }^{4}$ le proporcionó con el propio Julian Steward. Los años que González pasó en esa prestigiosa universidad norteamericana fueron determinantes en su formación e indirectamente impactarían con fuerza en la renovación de ideas y prácticas en el campo antropológico local, tanto los postulados teóricos que Steward le inculcó a sus alumnos como las modernas técnicas de investigación arqueológica, que los estudiantes de Columbia concretaban en la Universidad de Arizona. Su contacto como estudiante de Steward en un seminario de etnografía americana resultó, según su propio relato:

"fundamental porque me hacía ver la importancia de comparar los distintos pueblos americanos conocidos por la etnografía y tratar de buscar explicaciones de tipo cultural; esto para una formación general más o menos amplia, no dogmática, tratar de juntar distintas ramas de las ciencias del hombre, de la antropología, para tener una visión integral de los pueblos. Creo que en mí eso repercutió profundamente y hasta ahora. La visión que he tenido de mi trabajo toda la vida obedece, en buena parte, a esa formación" (Bianciotti 2005: 171-2).

\footnotetext{
4 En algunas entrevistas que le realizaron, González no menciona la labor de intermediación de Serrano y suele explicar su decisión de emigrar a los Estados Unidos en el consejo que le habría formulado el antropólogo francés de origen suizo Alfred Métraux (1902-1963), que había trabajado durante una década en la Universidad de Tucumán (Bianciotti 2005). Los intercambios epistolares durante 1945 entre Steward y Serrano (a quien el norteamericano se refería como "querido amigo") muestran que en efecto Serrano lo había recomendado a González para estudiar en Columbia y que el propio Steward había iniciado gestiones para que se le otorgue una beca. Debo estos y otros documentos a Mirta Bonnín, directora del Museo de Antropología de Córdoba.
}

Como recuerdan los alumnos de González durante la década del sesenta, sus clases tenían a Theory of Culture Change. The Methodology of Multilinear Evolution como un eje central, como las otras dos figuras descollantes de aquella "restauración nomotética" (Harris 1997), Leslie White y Gordon Childe. Pese a los debates que Steward mantenía con ellos, sus alumnos se resistieron sistemáticamente a concebirlos como verdaderos antagonistas y los consideran como parte de un "triunvirato" (Peace 2008) verdaderamente revolucionario en el campo de la antropología. Por supuesto, esa "revolución" no fue bien acogida por el campo antropológico local que, liderado por José Imbelloni y Oswald Menghin, ${ }^{5}$ rechazaba las corrientes norteamericana y británica y se aferraba a la teoría alemana de los Kulturkreise. El mainstream argentino prefería recostarse sobre una visión humanística (propia del historicismo alemán) y era incompatible con las teorías que buscaban leyes y regularidades, como el neoevolucionismo y, particularmente, la ecología cultural de Steward.

Por el contrario, el objetivo explícito en la obra de Imbelloni consistía en "dar una idea general de la relación entre el hombre y la civilización" (Imbelloni 1935:23), en el marco de la cual definía cultura como "una entidad abstracta y potencial del espíritu de una sociedad humana organizada" (Imbelloni 1935: 33). Para Imbelloni, la cultura también aparece como "una entidad provista de vida autónoma, suficiente en relación a las necesidades humanas, internamente organizada y original en cuanto a la solución de los problemas de la vida material, social y mental del hombre" (Imbelloni 1935: 150). Este proyecto intelectual daba forma a una Etnología que para desarrollarse como síntesis de las culturas del mundo necesitaba los materiales de disciplinas como la Etnografía, la Lingüística, la Arqueología y la Musicología, entre las más importantes. Incluso aseguraba que "la Etnología es la ciencia de nuestro siglo, y hacia ella se orientan todos aquellos que desean penetrar a fondo las instituciones humanas, por ejemplo, el derecho. $\mathrm{O}$

\footnotetext{
5 Oswald Menghin nació en Austria (1888-1973) y desde su llegada a la Argentina en 1948 ejerció una notable influencia en el campo arqueológico argentino que se mantuvo inalterado por décadas. Los más firmes cuestionamientos que recibió están vinculados con las actuaciones políticas en su país natal, por las que fue considerado prisionero de guerra (liberado luego en 1947) tras la derrota alemana en la segunda guerra mundial, principalmente por su participación como Ministro de Cultura y Educación del gobierno pro-nazi de SeyssInquart en 1938. De todos modos, y aunque su compromiso tan directo con el nazismo fue relativizado (sobre todo por su militancia católica), Kohl y Pérez Gollán (2002) analizan en detalle su obra e interpretan la carrera de Menghin como una muestra de los extraordinarios "peligros de combinar política, religión y prehistoria" (Kohl y Pérez Gollán 2002: 561). Por ello, la trayectoria académica de Menghin "ilustra los peligros de enfatizar la relevancia del conocimiento de un especialista de prehistoria para resolver problemas políticos y sociales contemporáneos" (Kohl y Pérez Gollán 2002: 562), del mismo modo que su "rigidez ideológica lo llevó a involucrarse en actividades políticas censurables y a distorsionar e interpretar tendenciosamente los registros etnográficos" (Kohl y Pérez Gollán 2002: 562).
} 
las directrices esenciales de nuestra mente: religión, arte y filosofía" (Imbelloni 1935: 43). Dentro del proyecto global de la Etnología, el autor italiano desarrolló el "neologismo" de Culturología a la que definía como:

"la ciencia que estudia la vida de las Culturas, como producto de la actividad de las sociedades humanas. La palabra «vida» comprende ya, claramente, tanto el concepto de formación y desarrollo, como el de difusión, predominio y sucesión; en una palabra, todas las etapas de su proceso histórico. Por otra parte, en la idea de Cultura están concebidos, en su totalidad, los elementos patrimoniales del hombre. Luego, son partes analíticas de la Culturología: la historia de la economía, de la técnica, de la religión, del arte, del estado, etc." (Imbelloni 1935: 182-183).

En definitiva, los postulados teóricos que -aunque contemplaban los fenómenos de difusión culturalprestaban especial atención a los condicionamientos medioambientales, a la identificación de tipos culturales y áreas culturales, al desarrollo tecnológico de las sociedades y que privilegiaban la construcción de cronologías absolutas, no podían encajar en la propuesta de Culturología y en las diversas utilizaciones del difusionismo alemán. De hecho, los primeros trabajos de González no contienen demasiadas referencias teóricas que busquen polemizar con el mainstream dominante, mucho más cuando se trataba de publicaciones gestadas en los espacios institucionales controlados por Imbelloni. No por ello, esas producciones se privan de formular observaciones críticas a las ideas dominantes en el campo antropológico local, en especial las metodológicas. Por ejemplo, un artículo publicado en Runa indicaba que:

"No es imposible que algún día podamos vincular específicamente algunos de los antiguos horizontes de cazadores de América del Norte con sus similares de América del Sud y Mesoamérica y elaborar secuencias válidas de gran amplitud geográfica, pero debido a la enorme variedad y a la diversidad tipológica de los instrumentos utilizados habrá que tener mucha cautela en la valoración de estas afinidades" (González 1952: 129).

En ese mismo artículo prefiguraba que "la etapa de investigación inevitable que se nos impone es la de tratar de hallar y definir los grandes complejos dentro de las distintas áreas, para lo que se requiere mucha y cuidadosa labor en el terreno, tarea más que olvidada entre nosotros" (González 1952: 130).

González se inició en la docencia en la Universidad Nacional de La Plata en 1949 (Soprano 2006) en la cátedra de arqueología que estaba a cargo de Enrique Palavecino. ${ }^{6}$ Muy poco tiempo después, ya entrada la

\footnotetext{
${ }^{6}$ González identifica a Palavecino, junto con Serrano y Vignati, como arqueólogos de la vieja guardia que "concentraban su actividad en
}

década del cincuenta, Serrano le ofreció hacerse cargo de la cátedra de Arqueología Argentina de la Facultad de Filosofía y Letras de la Universidad del Litoral en Rosario, ${ }^{7}$ que él mismo dictaba y que había dejado vacante para enseñar en la Universidad de Córdoba. Según sus propios testimonios, González se vio obligado allí a perfeccionar sus lecturas y ofrecer un panorama teórico más compacto para sostener ambas cátedras (Bianciotti 2005), lo que sería sustancial en la formación de futuros arqueólogos y antropólogos sociales. Desde sus cátedras ${ }^{8}$ intentó recalcar "la necesidad de que el arqueólogo se proveyera de una sólida formación en teoría de la cultura. En líneas generales enfatizamos, hacia aquella década, las propuestas formuladas por Gordon Childe" (González 1991-1992: 102).

La influencia de la obra de Steward en la enseñanza de la antropología argentina puede apreciarse también a través de la labor de aquellos alumnos y discípulos de de González que se hicieron cargo de cátedras que él dictaba o que él mismo promovió. El caso más evidente tal vez sea el de José Cruz, el discípulo propuesto por González para dictar las materias de antropología social/cultural en las instituciones en las que pudo concretar un mayor peso institucional. Cruz era un graduado de Historia (con orientación en antropología) de Córdoba que además había participado en los trabajos de campaña arqueológicos encabezados por González, puntualmente en Laguna Blanca (Catamarca). Cruz enseñaba "Antropología Cultural" en la Facultad deFilosofía y Humanidades de la Universidad Nacional de Córdoba y la misma materia, denominada Antropología Social (optativa de la carrera de Antropología), en la Facultad de Ciencias Naturales y Museo de la Universidad Nacional de La Plata. Como consta en el programa de 1965 de Antropología Cultural en Córdoba, Cruz planificó una materia con una densa carga de contenidos que ofrecía un panorama actualizado de la disciplina en la que predominaban ampliamente los autores norteamericanos. Y por supuesto, la obra de Steward y otros materialistas, como White, ocupaba una porción significativa de esos contenidos. En la primera parte del programa (dividida a su vez en tres unidades) Cruz planteaba una "aproximación

trabajos de campaña o de etnohistoria" (González 1991-92: 93). Salvo a Palavecino, los consideraba desactualizados en la moderna teoría antropológica. Precisamente, Palavecino era uno de los dos profesores (junto con Ciro René Lafón) que enseñaron a Julian Steward en la carrera de antropología de la Universidad de Buenos Aires en la década del sesenta.

7 El Instituto de Antropología de la Universidad Nacional de Litoral fue creado en 1951 en Rosario y comenzó a funcionar un año más tarde. Desde su creación fue dirigido por Antonio Serrano, siendo luego reemplazado por Alberto Rex González. Garbulsky (2004) señala que la reforma del plan de estudios de la carrera de historia en 1957 (y que entró en vigencia en 1959) permitió la creación de una orientación en antropología, en la que González tuvo una activa participación.

8 Por ejemplo, en la carrera de La Plata, González dictó durante la década de 1960 en los sucesivos planes materias como Arqueología Americana (culturas pre-cerámicas)", "Arqueología Americana (culturas agro-alfareras)", Prehistoria General y Arqueología Argentina. 
epistemológica" de la disciplina, en la que se advierte una exposición ecléctica de autores (Schobinger, EvansPritchard, Beals, Kluckhohn, Herskovitz y Haddon) que no excluía a Imbelloni con su Epítome de Culturología, lo que completaba un panorama amplio de las distintas tendencias y subdisciplinas de la ciencia antropológica, que implicaba al "problema terminológico". La línea epistemológica se continuaba en la segunda unidad, en donde ya se comienza a advertir una toma de posición teórica en la elección de los autores y las problemáticas, como la relación de la antropología con la historia y en general con las diversas "ciencias del hombre". El perfil de la subdisciplina de la antropología cultural se define con mayor claridad en la tercera unidad ("La practicidad de la antropología"). En ese segmento del programa ya se postulan las problemáticas contemporáneas y hasta se llega a plantear una noción de gran relevancia en la época (y mucho más todavía en los años venideros), como la de compromiso. ${ }^{9}$ Directamente ligado a ello, se consideraban la antropología aplicada, la problemática del cambio dirigido y los estudios de desarrollo. Se destacan en esa parte autores como Sol Tax, Raymond Firth y George Foster.

La segunda parte del programa ("bolillas"10 4 y 5), denominada "Aproximación metodológica", consideraba los métodos etnográfico, comparativo y genealógico, además de ocuparse puntualmente de la vinculación de la antropología con la sociología. En los aspectos específicamente referidos al método etnográfico se analizaban problemáticas tales como la "aceptación, identificación y adaptación", el "uso de idiomas indígenas", el "informante clave" y los "estudios de áreas culturales", todas ellas de histórico peso en la etnografía norteamericana. Ya en esta parte comienzan a aparecer trabajos de Steward como referencias centrales, aunque siempre en medio una gran cantidad de culturalistas norteamericanos, lo que queda muy claro en la sexta unidad ("La Cultura") en la que predominan antropólogos como Kluckhohn, Kroeber, Lowie, Beals y Herskovitz. Será en la séptima "bolilla" ("Dinámica e interpretación de la cultura") cuando pueda notarse con mayor claridad la influencia de Steward, no sólo por los textos de lectura obligatoria sino por el enfoque propuesto, en donde se destacan los integrantes de aquel mencionado "triunvirato" materialista y neoevolucionista: Steward, Childe y White. Allí es donde se postula la

9 La noción de compromiso adquirió en los años sesenta una importancia vital en las representaciones de los cientistas sociales, cada vez más vinculados con la política, lo que daría una notable relevancia a la figura del intelectual comprometido, frente al experto del período desarrollista y al anterior intelectual humanista (Suasnábar 2004; Gil 2010). Anclada en la concepción sartreana, comenzó a ligarse cada vez más en América Latina con los proyectos revolucionarios a los que muchos de sus componentes adherían en ciertos casos de forma explícita, en un contexto en el que "la revolución fue para muchos ciudadanos y dirigentes una declaración de principios" (Ollier 2005: 10). Guber (2008) se ha ocupado detalladamente de cómo se utilizó esa categoría en la antropología social argentina.

${ }^{10}$ Con ese término aparecen segmentados los contenidos. problemática del cambio cultural, se retoma el concepto clásico de evolución para luego sí focalizar en las nuevas formulaciones teóricas sobre ese mismo concepto, además de considerar especialmente la ecología cultural y "su aplicación al origen de las primeras culturas urbanas". Steward adquiere en esta parte del programa una significativa importancia, configurándose -dentro de un grupo de autores relevantes en la disciplina- como una de las referencias privilegiadas. Las restantes otras "bolillas" también descansaban sobre el arsenal teórico de la antropología norteamericana, ${ }^{11}$ y estaban dedicadas a la Escuela de Cultura y Personalidad, "La Familia", "Las instituciones políticas y económicas", "La religión en la sociedad ágrafa" y "La aculturación".

El programa de la materia que el propio Cruz dictaba el mismo año en La Plata estaba estructurado sobre principios similares pero no era exactamente igual. Constaba de siete unidades y era algo más restringido y específico de antropología social. Esa asignatura dictada en La Plata también contenía una proporción alta de autores antropológicos norteamericanos, aunque también agregaba algunos referentes de la sociología de ese país, como Talcott Parsons y Robert Merton. La primera unidad, de contenido epistemológico, se posicionaba sobre densas problemáticas disciplinares, como el objeto de estudio, sus principales categorías de análisis y retomaba, entre otros aspectos, la noción de compromiso del antropólogo. También se desarrollaba en este segmento el contexto histórico del surgimiento y el desarrollo de la antropología social. Los enfoques materialistas y neoevolucionistas (Steward, White y Childe) aparecían en la parte final de la segunda unidad ("Aproximación Gnoseológica"), denominada "Estructura y Cultura". Steward ${ }^{12}$ volvía ser referenciado en la tercera unidad ("aproximación metodológica"). Precisamente, como muestra Soprano (en este volumen) en un análisis detallado de los programas y la labor institucional de actores claves en el escenario antropológico platense,

\footnotetext{
11 Por supuesto, entre las citas dominantes se destacan varios de los ya nombrados, juntos con otros de gran relevancia en la tradición norteamericana (Lowie, Kroeber, Linton, Redfield, Mead), pero también aparecían, siempre en menor cantidad, de otras tradiciones, como Firth, Malinowski (los británicos más referidos), Frazer (para la parte de "magia") y -sobre todo en las primeras unidades- LéviStrauss.

12 Una de las tantas características de la obra de Steward es que nunca hizo un trabajo de campo intensivo, sino que concretó estadías cortas desde una perspectiva naturalista y empirista. Sus trabajos en el terreno estaban enfocados en obtener "información confiable" y nunca le prestó demasiada atención -tal cual era costumbre en la antropología norteamericana de su época- a las formas de interacción con los nativos. Trabajaba prioritariamente con entrevistas a informantes en el marco de un trabajo intensivo con unas pocas fuentes, aunque con un énfasis marcado en la observación del paisaje y de los recursos tecnoambientales. Y además, le preocupaban los patrones de la conducta y la actividad social directamente observable. Las carencias analíticas de su trabajo de campo quedarían expuestas más tarde en el proyecto Puerto Rico, cuando sus estudiantes se vieron obligados a construir sus herramientas metodológicas durante el mismo proceder del trabajo de campo. Steward tampoco era demasiado sensible a considerar las denominaciones emic.
} 
Cruz ponía un marcado énfasis en la investigación sobre el terreno, para el cual había planificado en 1965 una serie de "reuniones de seminario preparatorio para los trabajos de campaña", orientados hacia el análisis de comunidades (como el que había hecho en Laguna Blanca con el equipo de Rex González), pero también había planificado ejercicios de etnografía urbana orientados hacia "los barrios de emergencia", concebidos para "preparar los diseños de investigación previos a la labor de campo que se cumplirán en el período de receso de la actividad docente". Lo restante del contenido de la asignatura (unidades denominadas "partes especiales") estaba referido a los estudios de comunidades, los sistemas de parentesco, la aculturación y la antropología aplicada.

Una instancia que podría haberse transformado en un punto de inflexión en la enseñanza y práctica de la antropología social es la materia de ese nombre (obligatoria para los alumnos de ciencias antropológicas de la UBA) que dictó en 1962 el destacado antropólogo norteamericano Ralph Beals, quien se desempeñó como profesor visitante en el departamento de Sociología. En aquellos años, merced a los subsidios internacionales que la gestión encabezada por Gino Germani obtuvo de fundaciones filantrópicas (Ford y Rockefeller) y de la UNESCO, fueron muchas las destacadas figuras de las ciencias sociales metropolitanas (Cicourel, Balan, Touraine, Silvert, Horowitz, Heintz, entre otros) que formaron parte del plantel docente de Sociología en la Facultad de Filosofía y Letras. Un antropólogo que aún no había iniciado la carrera en 1962 definió a aquella materia como "una leyenda"13 y no recuerda con precisión a compañero suyo de los años superiores que la hubiera cursado. Más allá de la mayor o menor presencia de los estudiantes de antropología en las clases de Beals, no se ha detectado vínculo posterior o influencia alguna declarada por antropólogos locales. ${ }^{14}$ Beals había sido compañero de Steward en Berkeley y luego siguieron teniendo estrechos vínculos personales y profesionales. En la materia que dictaba en la UBA, Beals apeló a numerosas referencias de Steward, sobre todo en la segunda parte

\footnotetext{
${ }^{13}$ Comunicación personal.

14 Es importante resaltar que, en los primeros años de la carrera de ciencias antropológicas, el Museo Etnográfico de Buenos Aires fue el escenario central de una vida intelectual intensa, a partir de las clases y conferencias que allí se impartían, pero también por los materiales que había a disposición, como colecciones, cajas, estantes y documentos que favorecían un contacto continuo entre profesores y alumnos. Como espacio propio y claramente separado de las aulas de la facultad, era el punto de encuentro predilecto y distintivo del ambiente antropológico porteño (Guber 2006). Aquella "unidad inicial" (Guber 2006) de profesores y alumnos en "El museo" se manifestaba especialmente frente a los otros estudiantes de la Facultad de Filosofía y Letras y a los demás estudiantes de antropología en el resto del país. Inclusive desde sociología, uno de los estigmas que solía proyectarse sobre la carrera de antropología era "cueva de fascistas" (Guber 2006). Sin embargo, luego de una primera etapa de plena identificación de estudiantes y profesores, aglutinados por el ámbito común y "sagrado" del Museo aquellos primeros graduados comenzaron a alejarse de sus maestros a partir de una serie de enfrentamientos disciplinares y políticos (Guber y Visacovsky 1999; 2000).
}

del programa, bajo los "ítems" referidos a los "sistemas ecológicos", a "las teorías y conceptos evolucionistas", a "las culturas pre-neolíticas", y las "culturas neolíticas y postneolíticas". Otros autores citados eran White, Nadel, Manners, Redfield y Lewis. Uno de los últimos ítems del segundo y último segmento del programa, denominado "el estudio de las culturas modernas y el carácter nacional", también contaba con referencias de Steward, además de, por supuesto, Ruth Benedict y Margaret Mead.

Por supuesto, los canales de difusión que se han mencionado no pueden presentarse como las únicas vías de acceso en el campo antropológico argentino de la obra de un autor descollante en la historia de la antropología del siglo XX. Sí tal vez fueron las más evidentes, principalmente a través de las cátedras y la formación de recursos en investigación de campo. Más allá del real impacto que el método de la ecología cultural y sus consiguientes enfoques neoevolucionistas haya producido entre los diversos actores del campo local, significó un aporte altamente contrastante con el tipo de antropología que, a grandes rasgos, se enseñaba y practicaba en la Argentina.

\section{Conclusiones}

La antropología argentina comenzó a experimentar transformaciones sustanciales en la segunda mitad del siglo $X X$, vinculadas con cambios relevantes en la estructura de funcionamiento de un campo disciplinar que estaba atravesando su definitiva institucionalización. Pero esas transformaciones transitaron caminos con ritmos marcadamente diferentes en las distintas subdisciplinas. Mientras que la antropología social encontró serios obstáculos (institucionales, de liderazgos, etc.) para poder desarrollarse, la arqueología experimentó mayores posibilidades de "progreso" alternativo a los lineamientos dominantes impuestos por el Kulturhistorische Methode en las carreras de las universidades centrales de Buenos Aires y La Plata. La renovación metodológica y teórica -no exclusiva por supuesto- que propició el liderazgo de Alberto Rex González a partir de su formación en los Estados Unidos impulsó la introducción de nuevos autores, en especial desde su tarea como formador en docencia en distintas universidades, pero también en su papel de "maestro informal" que le reconocen muchos de sus "discípulos" indirectos. Lo mismo le corresponde a los grupos de investigación que se formaron alrededor suyo. Así, la antropología experimentó un flujo de ideas por distintos canales, ya sea institucionales o parainstitucionales, que progresivamente fueron impactando en otros referentes locales del campo formados en otra matriz disciplinar. De ese modo, en el marco de la hegemonía que el difusionismo alemán siguió detentando en los espacios institucionales de Buenos Aires y La Plata, otros espacios de formación se continuaron abriendo también con otros referentes 
(por ejemplo Augusto Cortazar, Enrique Palavecino, Ciro Lafón, ${ }^{15}$ Susana Chertudi) que progresivamente fueron difundiendo en sus cátedras las nuevas ideas y enfoques que se imponían en el campo antropológico mundial.

Claramente se ha presentado aquí un relato vinculado con las estrategias de posicionamiento del propio González y de sus intérpretes autorizados. El análisis detallado de su obra, como de las huellas discursivas más puntuales de sus posicionamientos teóricos requiere una investigación mucho más detallada y de largo alcance. Por ello, en este artículo se ha presentado uno de los modos en los que impactó en la Argentina un clima intelectual preciso que en un espacio de origen también propició una importante renovación teórico-metodológica. En ese sentido, Steward es un autor clave en el desarrollo de la antropología, tanto en su país como en la Argentina. Su obra, gestada en un marco culturalista con el que nunca dejó de estar vinculado, se fue posicionando cada vez hacia posturas materialistas y neoevolucionistas. Sin embargo, ello no impidió que muchos de los alumnos que se nuclearon en torno a su liderazgo y fueron directamente influenciados por él, desarrollaran importantes trayectorias académicas en las que incorporaron herramientas analíticas más propias de la tradición británica, muy firmes en la Universidad de Chicago, institución en la que además se formaría Esther Hermitte, ${ }^{16}$ otro referente importante en la renovación de la antropología argentina. Allí, investigadores que participaron del Proyecto Puerto Rico como Eric Wolf (y algunos otros en instituciones diferentes, como por ejemplo Sidney Mintz desde Yale) llevarían adelante trabajos que de algún modo sintetizarían la tradición británica de trabajo de campo con los enfoques materialistas y de áreas culturales en los que se formaron. Precisamente, los estudios sobre campesinado

\footnotetext{
${ }^{15}$ El arqueólogo Ciro Lafón, aunque formado en el marco históricocultural y discípulo orgulloso de Imbelloni, estimuló el desarrollo de la antropología social en la UBA a la que consideraba un "un signo de modernización y nacionalización que no implicaba negar las ramas «clásicas», ni introducir una bandera extra académica, esto es, política" (Guber 2007; Visacovsky et al. 1997). Inclusive en los años setenta se pronunció favorable a "una antropología al servicio del país, de sus hombres y de sus instituciones" (Lafón 1974: 313). Además de la condición militante que implicaba proporcionar conocimiento para "dar testimonio de la situación real en la que estamos viviendo" (Lafón 1974: 313), Lafón aseguraba estar "intentando iniciar una Antropología Nacional, no dependiente, empezando por ensayar la elaboración de nuestro propio modelo. Que es COMPROMETIDO CON NUESTRO PAís" (Lafón 1974: 332).

16 Hermitte regresó de Chicago en 1965 y encontraría serias dificultades para insertarse en la estructura de enseñanza de las universidades rioplatenses. En la primera parte de 1966 tuvo a su cargo en la carrera de ciencias antropológicas de la UBA un seminario sobre "etnografía de los «mayas contemporáneos» con el mismo enfoque estructuralfuncionalista que había aplicado en su tesis doctoral" (Guber 2007: 281). Las posibilidades de esta innovación se cortaron con las renuncias masivas en la UBA luego de la Noche de los Bastones Largos a las que se plegó Hermitte, a partir de lo cual continuaría su labor en el Centro de Investigaciones Sociales (CIS) del Instituto Di Tella, un organismo privado que resultó central en el proceso de modernización cultural que atravesó la Argentina de la época y en el estímulo de la investigación en ciencias sociales según los parámetros internacionales.
}

latinoamericano se constituirían en referencia ineludible de los futuros "antropólogos sociales" de fines de los años sesenta y principios de los años setenta en la Argentina (Guber y Visacovsky 2000). De ese modo, el contexto periférico de la antropología argentina que estaba extremadamente ligado a una tradición metropolitana en retirada, comenzó a experimentar una mayor cercanía con otras tradiciones con mayor vigor en el campo antropológico mundial. Pero ese contacto fue extremadamente fragmentado (por ejemplo en lo institucional, lo geográfico) ya que además las sucesivas interrupciones institucionales cortaron procesos (como ocurrió en 1966 a partir de la Noche de los Bastones Largos) de una renovación más incipiente, sobre todo en el espacio institucional clave de la Universidad de Buenos Aires (Guber 2007). Esas limitaciones que iban surgiendo de los avatares de las política nacional se irían haciendo cada vez más determinantes en la década del setenta, cuando distintos proyectos académicos y trayectorias profesionales encontrarían obstáculos todavía muchos más serios que los que se habían producido con anterioridad. Pero esa es otra historia.

\section{Bibliografía}

Mar del Plata, agosto de 2010

Altamirano, C. 2008. Introducción general. En C. Altamirano (director) Historia de los intelectuales en América Latina I. la ciudad letrada, de la conquista al modernismo: pp. 9-27, Katz, Buenos Aires.

Bennett, Wendell; E.Bleiler y F.Sommer. 1948. Northwest Argentine Archaeology. Yale University Publications in Anthropology 38, New Haven.

Bianciotti, A. 2005. Alberto Rex González: la imagen y el espejo. Arqueología Suramericana 1(2):155-184.

Bourdieu, P. 2000 Intelectuales, política y poder, Buenos Aires, Eudeba.

Bourdieu, P. 2007. El sentido práctico, Siglo XXI, Buenos Aires.

Bourdieu, P. 2008. Homo academicus, Siglo XXI, Buenos Aires.

Boschin, M. T. 1998. Introducción. En AA. VV., Homenaje a Alberto Rex González. 50 años de aportes al desarrollo y consolidación de la antropología argentina: pp. 11-15, Facultad de Filosofía y Letras, UBA, Buenos Aires.

Carbonari, J. y A. Figini. 1998. Quien sabe lo que busca, interpreta lo que encuentra. Sobre contexto, secuencia y cronología en la obra de Alberto Rex González. En AA. VV., Homenaje a Alberto Rex González. 50 años de aportes al desarrollo y consolidación de la antropología argentina: pp. 75-81. 
Cardoso de Oliveira, R. 1995. Notas sobre una estilística da antropología. En R. Cardoso de Oliveira y G. Ruben (orgs.) Estilos de antropología, Editora da Unicamp, Campinas.

Ceballos, R. y C. Hidalgo. 1998. Alberto Rex González: la búsqueda de una interpretación integral de la cultura. En AA. VV., Homenaje a Alberto Rex González. 50 años de aportes al desarrollo y consolidación de la antropología argentina: pp. 19-23.

Clemmer, R. y L. D Myers. 1999. Introduction. En R. Clemmer; L. D. Myers y M. E. Rudden (eds.) Julian Steward and the Great Basin. The Making of an Anthropologist: pp. ix-xxii, University of Utah Press, Salt Lake City.

Darnell, R. 2001. Invisible Genealogies: A History of Americanist Anthropology, University of Nebraska Press, Lincoln.

Darnell, R. y S.Murray. 2001. Series editors's Introduction. En Invisible Genealogies: A History of Americanist Anthropology, University of Nebraska Press, Lincoln.

Garbulsky, E. 2004. La Producción del Conocimiento Antropológico-Social en la Facultad de Filosofía y Letras de la Universidad Nacional del Litoral, entre 19551966. Vínculos y relaciones nacionales. Cuadernos de Antropología Social, 20: 41-60.

Gil, G. J. 2010. Periferia, militancia revolucionaria y transformación de la sociedad. Un estilo antropológico en los sesenta y los setenta en la Argentina. En Gastón Julián Gil (ed.) Universidad y utopía. Ciencias sociales y militancia en la Argentina de los 60 y 70: pp. 145-198, EUDEM, Mar del Plata.

González, A. R. 1952. Antiguo horizonte precerámico en las Sierras Centrales de la Argentina. Runa, V: 110-133.

González, A. R. 1974. Arte, estructura y arqueología, Nueva Visión, Buenos Aires.

González, A. R. 1998. El rol de los sistemas simbólicos en el proceso de evolución cultural. En AA. VV., Homenaje a Alberto Rex González. 50 años de aportes al desarrollo y consolidación de la antropología argentina: pp. 363-378.

González, A. R. y Pérez Gollán, J. A. 1972. Argentina Indígena. Vísperas de la conquista, Paidós, Buenos Aires.

Guber, R. 2006. Linajes ocultos en los orígenes de la antropología social de Buenos Aires. Avá, 8: 26-55.

Guber, R. 2007. Crisis de presencia, universidad y política en el nacimiento de la antropología social de Buenos Aires, Argentina. Revista Colombiana de Antropología, 43: 263-298.
Guber, R. 2008 Antropólogos-ciudadanos (comprometidos) en la Argentina. Las dos caras de la "antropología social" en 1960-1970. Journal of the World Anthropology Network, 3: 67-109.

Guber, R. y S. Visacovsky. 1999. Controversias filiales: la imposibilidad genealógica de la antropología social de Buenos Aires. Relaciones de la Sociedad Argentina de Antropología, XXII-XXIII: 25-53.

Guber, R. y S. Visacovsky. 2000. Nación, marginalidad crítica y el Otro interno en la antropología social argentina de los 1960s-70s. Desarrollo Económico. Revista de Ciencias Sociales, 40 (158): 289-316.

Harris, M. 1997. El desarrollo de la teoría antropológica. Una historia de las teorías de la cultura, Siglo XXI, México.

Imbelloni, José. 1935. Epítome de Culturología, Humanior, Buenos Aires.

Kerns, V. 2003. Scenes from the High Desert. Julian Steward's Life and Theory, University of Illinois Press, Urbana and Chicago.

Kohl, P. y J. A. Pérez Gollán. 2002. Religion, Politics, and Prehistory. Reassessing the Lingering Legacy of Oswald Menghin. Current Anthropology, 43 (4): 561-586.

Lafón, C. 1974. Un modelo no tradicional para el estudio del cambio cultural en territorio argentino del siglo XVI en adelante. Relaciones, 8: 311-337.

Llamazares, A. 1998. Aportes de Alberto Rex González al estudio del arte precolombino. En AA. VV., Homenaje a Alberto Rex González. 50 años de aportes al desarrollo y consolidación de la antropología argentina: pp. 61-73.

Márquez Miranda, F. 1958. Pueblos y culturas de América, Editorial Nova, Buenos Aires.

Ollier, M. 2005. Golpe o revolución. La violencia legitimada, Argentina 1966/1973, Eduntref, Buenos Aires.

Patterson, T. y A. Lauria-Perricelli. 2000. Julian Steward and the Construction of Area-Studies Research in the United States. En R. Clemmer; L. D. Myers y M. E. Rudden (eds.) Julian Steward and the Great Basin. The Making of an Anthropologist. University of Utah Press, Salt Lake City.

Peace, W. 2008. Columbia University and the Mundial Upheaval Society: A Study in Academic Networking. En D. Wax (ed.) Anthropology at the Dawn of the Cold War: pp. 143-165, Pluto Press, London.

Pérez Gollán, J. A. 1981. Presencia de Vere Gordon Childe, Instituto Nacional de Antropología e Historia, México. 
Pérez Gollán, J. A. 1998. La presencia de Alberto Rex González en la Universidad de Córdoba. Estudios, 10: 17-29.

Politis, G. 2001. On Archaeological Praxis, Gender Bias and Indigenous Peoples in South America. Journal of Social Archaeology 1(1): 90-107.

Politis, G. 2005. Los aportes de Guillermo Madrazo a la arqueología pampeana. Andes 16: 93-117.

Ratier, H. 1998. Alberto Rex González y la Antropología Social en la Argentina. En AA. VV., Homenaje a Alberto Rex González. 50 años de aportes al desarrollo y consolidación de la antropología argentina: pp. 39-46.

Ringuelet, R. 1998. Antropología social y Arqueología. En AA. VV., Homenaje a Alberto Rex González. 50 años de aportes al desarrollo y consolidación de la antropología argentina: pp. 47-60.
Soprano, G. 2006. Continuidad y cambio en los estudios en etnología de poblaciones indígenas contemporáneas y comunidades folk en la facultad de ciencias naturales y museo de la Universidad nacional de la Plata. Anuario de Estudios en Antropología Social, 3: 23-51.

Steward, J. H. 1972. Theory of Culture Change. The Methodology of Multilinear Evolution, University of Illinois Press, Urbana and Chicago.

Suasnábar, C. 2004. Universidad e intelectuales. Educación y política en la Argentina (1955-1976), FLACSO-Manantial, Buenos Aires.

Visacovsky, S. y R. Guber. 1997. Modernidad y tradición en el origen de la carrera de Ciencias antropológicas de la Universidad de Buenos Aires. Redes, 10: 213-257.

Wolf, E. 2006. Europa y la gente sin historia, Fondo de Cultura Económica, México. 